\title{
Table of statutes and statutory instruments
}

Paragraph numbers in bold indicate a mention in the text: paragraph numbers in roman type indicate a mention only in a footnote to text in that paragraph.
Assize of Bread and Ale $1266 \quad \mathbf{6 . 3 4}$
Commercial Agents (Council
Directive) Regulations
19937.35
Consumer Credit Act 1974

$$
6.08
$$

Schedule $4 \quad \mathbf{6 . 0 8}$

Housing Act 1961

s.32 3.25, 3.27, 3.28, 3.29, 3.36

Law Reform (Married Women and Tortfeasors) Act 1935 3.15, 3.16

Leeman's Act 1857 (30 \& 31 Vict. c. 29) 5.20

Marine Insurance Act 1906 6.57

s. $25 \quad 6.62$

s.33 $\mathbf{6 . 5 7}$

s.34 $\mathbf{6 . 5 8}$

s.36 $\mathbf{6 . 5 9 ,} \mathbf{6 . 6 9 , 6 . 7 0}$

s.39 $\mathbf{6 . 6 0 ,} \mathbf{6 . 6 5}$

s. $40 \quad \mathbf{6 . 6 0}, \mathbf{6 . 6 1}, \mathbf{6 . 6 6}$

s.41 6.60, 6.67

Occupier's Liability Act $1957 \quad 3.03$

Road Traffic Act $1930 \quad \mathbf{3 . 1 4}, \mathbf{3 . 1 8}$ Sale of Goods Act 1979 (includes reference to $1893 \mathrm{Act}) \mathbf{1 . 0 1}$,
$1.04,2.22,6.01,6.03,6.10,6.23$

6.73

s. $2 \quad \mathbf{6 . 1 5}$

s.12 1.04, 1.11, 1.32, 6.08, 6.12ff, $6.55,6.56,6.74$

s.13 1.04, 1.11, 6.08, 6.22, 6.26ff, $6.55,6.56,6.74$

s.14 1.04, 1.11, 1.20, 5.37, 6.08, 6.30, 6.36ff, 6.54, 6.55, 6.56, $6.73,6.47$

s. $151.04,1.11,6.08,6.52 \mathrm{ff}, 6.55$, 6.56, 6.74

s.15A 6.31

s. $17 \quad \mathbf{1 . 3 0}, \mathbf{1 . 3 1}, \mathbf{6 . 0 8}$

s.18 1.30, 1.31, 6.08

s.30 $\quad \mathbf{6 . 2 9}$

Sale and Supply of Goods Act $1994 \quad 6.31$

Statute Law Revision Act $1863 \quad 6.34$

Supply of Goods and Services Act $19826.01, \mathbf{6 . 0 3}$

s.2 6.08, 6.14, 6.19

s. $3 \quad \mathbf{6 . 0 8}$

s.4 $\mathbf{6 . 0 8 ,} 6.73$

s.5 $\mathbf{6 . 0 8}$

Supply of Goods (Implied Terms)

Act $1973 \mathbf{6 . 0 3 , 6 . 4 0 , 6 . 5 1}$

s. $8 \quad 6.08,6.14,6.20,6.56$ 
s.9 $\mathbf{6 . 0 8}$

s. $10 \quad 6.08,6.73$

s. 116.08
Unfair Contract Terms Act 1977 s.6 $1.04, \mathbf{6 . 2 1}, \mathbf{6 . 5 6}, \mathbf{6 . 7 4}$ 